\title{
Glaucoma patients demonstrate faulty autoregulation of ocular blood flow during posture change
}

\author{
David W Evans, Alon Harris, Melanie Garrett, Hak Sung Chung, Larry Kagemann
}

\begin{abstract}
Backgroundlaims-Autoregulation of blood flow during posture change is important to ensure consistent organ circulation. The purpose of this study was to compare the change in retrobulbar ocular blood flow in glaucoma patients with normal subjects during supine and upright posture.
\end{abstract}

Methods-20 open angle glaucoma patients and 20 normal subjects, similar in age and sex distribution, were evaluated. Blood pressure, intraocular pressure, and retrobulbar blood velocity were tested after 30 minutes of sitting and again after 30 minutes of lying. Retrobulbar haemodynamic measures of peak systolic velocity (PSV), end diastolic velocity (EDV), and resistance index (RI) were obtained in the ophthalmic and central retinal arteries using colour Doppler imaging (CDI). Results-When changing from the upright to supine posture, normal subjects demonstrated a significant increase in $\mathrm{OA}$ EDV $(p=0.016)$ and significant decrease in OA RI ( $p=0.0006)$ and CRA RI ( $p=$ 0.016). Glaucoma patients demonstrated similar changes in OA measures of EDV $(p=0.02)$ and $R I(p=0.04)$, but no change in CRA measures.

Conclusion-Glaucoma patients exhibit faulty autoregulation of central retinal artery blood flow during posture change. (Br F Ophthalmol 1999;83:809-813)

Regulation of blood flow during posture change is important to maintain proper circulatory status in many organs. When in the upright posture, gravity pulls blood away from organs above the heart and towards organs below the heart. To ensure appropriate blood flow when changing from supine to upright posture, vessels below the heart constrict to block hyperaemia and oedema, while vessels above the heart dilate to offset falling perfusion pressure.

An abnormal change in blood flow during postural variation is an indication of vascular irregularity. For example, in the normal foot, vessels constrict when the foot is placed below the heart, causing blood flow to fall by $50 \%{ }^{1}$ However, in patients with lower limb arterial obstructive disease, blood flow increases by approximately $80 \%$ when the patient stands. In normal cerebral circulation, blood flow remains virtually constant during posture change. ${ }^{2}$ Middle cerebral artery blood velocity varies less than $10 \%$ in the supine compared with sitting positions. Patients with autonomic dysfunction, however, exhibit wide variation in cerebral blood flow during posture change. ${ }^{23}$ In these patients middle cerebral artery blood velocity falls by more than $25 \%$ when changing from a supine to upright posture. ${ }^{3}$

Numerous recent studies suggest that dysfunctional ocular circulation contributes to the pathogenesis of glaucoma. ${ }^{4-7}$ Few data are available concerning the autoregulatory response of ocular blood flow to posture change in glaucoma patients. Analysis of ocular blood flow during various body positions may provide insight into the circulatory irregularity of glaucoma patients. The purpose of this study was to measure and compare the ophthalmic and central artery haemodynamics in glaucoma patients and normal subjects in both the seated and supine positions.

\section{Subjects and methods}

Paid volunteers, comprising 20 glaucoma patients and 20 normal subjects, participated in this study. This study was approved by the Indiana University institutional review board and all participants reviewed and signed informed consent statements before entering the study. Subjects and patients were examined by members of the Indiana University Medical Center Department of Ophthalmology Glaucoma Service. Both groups were free of diabetes, cardiovascular or respiratory disease, and were taking no medications for systemic hypotension. All patients had glaucomatous type optic disc appearance and/or visual field defects. The patient's cup/disc ratios were all greater than 0.6 and the loss in visual field sensitivity (as determined by mean defect) ranged from -5.0 to $-20.0 \mathrm{~dB}$. No limitation was placed on the minimum level of patient intraocular pressure for study inclusion. The control group was selected to be similar for age, sex distribution, blood pressure, and heart rate to the patient group (Table 1). Normal subjects had no history of ophthalmic disorders and results of an ophthalmic examination were normal. If a patient was on a regimen of ocular hypertensive therapy for glaucoma, he or she ceased taking medication 4 weeks before the start of the experiment.

\section{Methods}

This study was performed as part of a larger study in which participants were admitted to the General Clinic Research Center (GCRC) of the Indiana University Hospital for over-
Accepted for publication 8 February 1999 
Table 1 Age, sex distribution, heart rate, systolic blood pressure, and diastolic blood pressure (mean (SD)) for the glaucoma patients and normal subjects

\begin{tabular}{llll}
\hline & Glaucoma & Normal & $p$ Value \\
\hline Age (years) & $56(12)$ & $58(11)$ & NS \\
Sex & $13 \mathrm{M} / 7 \mathrm{~F}$ & $10 \mathrm{M} / 10 \mathrm{~F}$ & $\mathrm{NS}$ \\
BP sys (mm Hg) & $132(20)$ & $131(21)$ & $\mathrm{NS}$ \\
BP dias (mm Hg) & $76(10)$ & $76(12)$ & $\mathrm{NS}$ \\
Heart rate (beats/min) & $76(14)$ & $74(10)$ & NS \\
\hline
\end{tabular}

night observation. Patients and subjects entered the CGRC at approximately $5 \mathrm{pm}$ and were assigned private rooms. They had been instructed not to smoke or partake of caffeine or alcohol after noon on the day of testing. Upon arrival, each participant was evaluated and admitted by the nursing staff of the GCRC. After the admission process, they were asked to sit in the chair supplied in each patient room, in an erect position, with feet on the floor for 30 minutes. At the end of the $30 \mathrm{~min}$ utes, the patients and subjects were tested for blood pressure/heart rate, intraocular pressure, and retrobulbar ocular blood velocity using colour Doppler imaging (CDI). The right arm and right eye were selected for measurement, except in the cases of two patients which had unilateral glaucoma of the left eye. In these two cases, the left eye and left arm were used for testing.

Upon completion of testing, the participants were instructed to lie in a supine position for 30 minutes. Patients and subjects were told not to sleep during this 30 minute period and periodic checks were made to ensure compliance with this instruction. At the end of this 30 minute period, participants were evaluated in the supine position using the same procedures and schedule as described for the sitting position.

COLOUR DOPPLER IMAGING

Retrobulbar blood flow velocity was assessed using CDI, an ultrasound technique that combines b-scan grey scale imaging of tissue structure, coloured representation of blood flow based on Doppler shifted frequencies, and pulsed Doppler measurement of blood flow velocities. A Siemens Quantum 2000 (Issaquah, WA, USA) with a $7.5 \mathrm{MHz}$ linear phase transducer was used. For this device, energy density is approximately $15 \mathrm{~mW} / \mathrm{cm}^{2}$ in the colour imaging mode, and approximately $77 \mathrm{~mW} / \mathrm{cm}^{2}$ during the spectral analysis mode. Measurements of peak systolic velocity (PSV) and end diastolic velocity (EDV) were taken of the ophthalmic (OA) and central retinal (CRA) arteries. To examine the ophthalmic artery, the sample volume (segment along the transmitted beam chosen for analysis, approxi-

Table 2 Results for glaucoma patients and normal subjects in sitting and supine positions (mean (SD))

\begin{tabular}{lllllll}
\hline & Sit & Supine & p Value & Sit & Supine & p Value \\
\hline HR & $76(14)^{\star}$ & $70(11)^{\star}$ & $0.0001^{\star}$ & $74(10)^{\star}$ & $69(9)^{\star}$ & $0.0001^{\star}$ \\
BP sys & $132(20)$ & $130(17)$ & & $131(21)$ & $130(23)$ & \\
BP dias & $76(10)^{\star}$ & $73(9)^{\star}$ & $0.028^{\star}$ & $76(12)^{\star}$ & $71(9)^{\star}$ & $0.002^{\star}$ \\
IOP & $18.4(4) \#$ & $17.9(3) \# \#$ & & $13.7(3) \#$ & $13.9(3) \# \#$ & $0.0009 \#$ \\
& & & & & & $0.0004 \# \#$
\end{tabular}

*Statistically significantly difference between sitting and supine position. \#Statistically significant difference between glaucoma patients and normal subjects. mately $1.2 \times 1.2 \mathrm{~mm}$ ) was oriented nasally and superior to the optic nerve, just lateral to and abutting the visible hyporeflective stripe representing the nerve. The central retinal artery was found at the anterior of the optic nerve and the sample volume was placed with its centre about $2 \mathrm{~mm}$ behind the surface of the disc. After measurement of the flow velocities, the resistance index (RI) was subsequently calculated [(PSV - EDV)/PSV] by computer for each vessel measured. Recent studies ${ }^{8}$ have demonstrated good reproducibility for CDI measurement of the vessels evaluated in this study.

\section{BLOOD PRESSURE/HEART RATE}

Blood pressure and heart rate were determined by an automated oscillatory device (Dinamap 1486, Critikon Inc, Tampa, FL, USA). Oscillatory blood pressure devices determine blood pressure by sensing the changes in the pressure wave as blood resumes in the brachial artery during cuff deflation. The cuff was placed on the arm of the participant and the measurement was manually initiated by the testing technician - that is, he or she pushed the start button. Staff members of the GCRC calibrated the Dinamap devices once a month against the standard clinical sphygmomanometry technique.

\section{INTRAOCULAR PRESSURE}

Intraocular pressure was determined using a hand held Markey-Marg tonometer (Tonopen, Biorad Inc). Two measurements were taken at each testing session. If the two measurements were more than $2 \mathrm{~mm} \mathrm{Hg}$ apart, a third measurement was taken. To test IOP, the eye was held open and one drop of proxymetacaine (proparacaine) anaesthetic was instilled onto the cornea. While still holding the eye open, the Tono-pen was touched to the eye several times during a 5-10 second time period. The device was touched to the eye at the centre of the cornea (that is, in the centre of the pupil) at an angle perpendicular to cornea. When an accurate measurement of IOP was obtained, the Tono-pen emitted a tone. For the second measurement, the device was reset and touched to the eye again several times as just described. The Tono-pen has an internal calibrating system. It was calibrated at the beginning of each test session.

\section{STATISTICAL ANALYSIS}

Paired Student's $t$ tests were used to assess changes in measures within groups between the seated and supine measurements. Unpaired Student's $t$ tests were used to compare the measurements between groups for the two different body positions. Bonferroni's correction was applied when multiple $t$ tests were performed using a single data set. A $p$ value of $<0.05$ was considered statistically significant.

\section{Results}

Glaucoma patients and normal subjects were similar for heart rate, systolic blood pressure, and diastolic blood pressure in both body posi- 

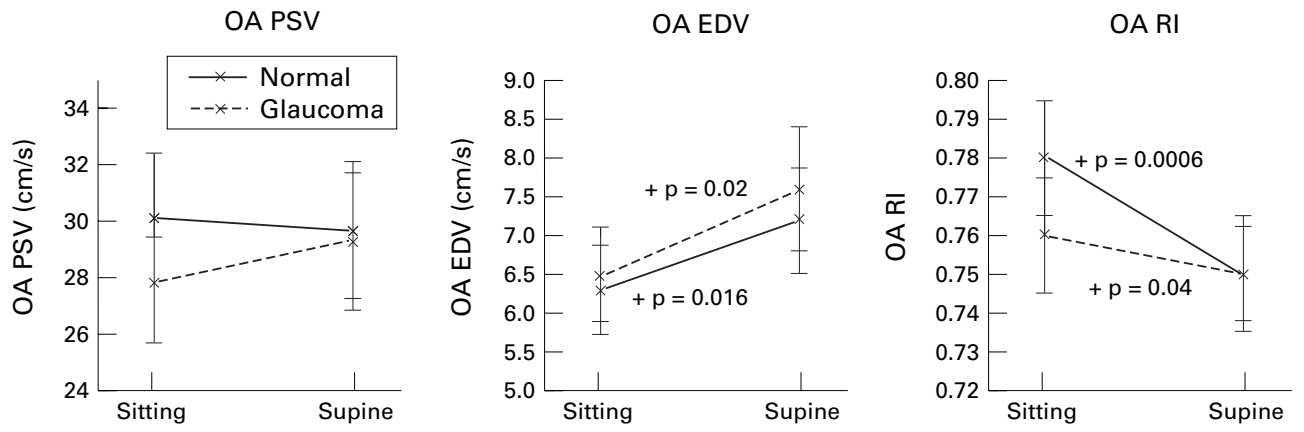

Figure 1 Peak systolic (PSV) and end diastolic blood flow (EDV) velocities and resistance index (RI) for the ophthalmic artery $(O A)$ in the sitting and supine position for glaucoma patients and normal subjects (mean (SEM)) The + indicates a significant change within groups from the sitting to supine posture.
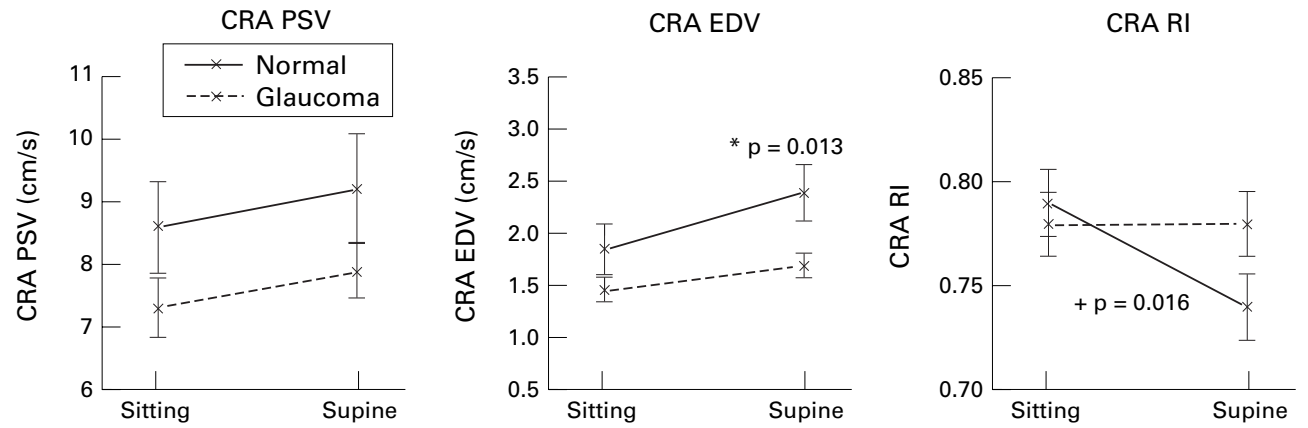

Figure 2 Peak systolic (PSV) and end diastolic blood flow (EDV) velocities and resistance index (RI) for central retinal artery (CRA) in the sitting and supine position for glaucoma patients and normal subjects (mean (SEM)). The + indicates a significant change within groups from the sitting to supine posture. The *indicates a significant difference between groups in either of the two body positions.

tions. The groups were significantly different for intraocular pressure in both the seated $(\mathrm{p}=$ $0.0009)$ and supine positions $(\mathrm{p}=0.0004)$ (Table 2).

During posture change, both groups showed a significant change in heart rate (glaucoma $\mathrm{p}$ $=0.0001$; normal $\mathrm{p}=0.0001$ ) and diastolic blood pressure (glaucoma $\mathrm{p}=0.028$; normal $\mathrm{p}$ $=0.002)$. Neither group demonstrated any change for systolic blood pressure or intraocular pressure (Table 2).

Glaucoma patients and normal subjects were similar for measures of retrobulbar haemodynamics while sitting. During supination, glaucoma patients displayed significantly lower central retinal artery (CRA) end diastolic velocity (EDV) $(\mathrm{p}=0.013)$ than normal subjects. When changing from a seated to supine body position, both groups exhibited an increase in ophthalmic artery (OA) EDV (glaucoma $\mathrm{p}=0.02$; normal subjects $\mathrm{p}=0.04$ ) and a significant decrease in OA resistance index (RI) (glaucoma $\mathrm{p}=0.04$; normal subjects $\mathrm{p}=0.0006$ ) (Fig 1). Neither group showed a change in central retinal artery PSV or EDV, while only normal subjects showed a change in CRA RI ( $p=0.016)($ Fig 2$)$.

\section{Discussion}

Glaucoma patients and normal subjects demonstrate similar changes in heart rate, IOP, diastolic blood pressure, and ophthalmic artery haemodynamics during posture change. The CRA RI response to posture variation is significantly different between groups; it falls significantly in normal subjects when changing from a seated to supine position, but shows no change in glaucoma patients.

Constant intraocular pressure during posture change is contrary to the findings of many previous studies (for review see $\mathrm{Kothe}^{10}$ ). Intraocular pressure has a well established circadian rhythm and is known to peak in the morning and fall during the day, reaching its lowest point in early evening before sleep. ${ }^{11}{ }^{12}$ Whereas previous studies were conducted much earlier in the day, during a time period close to the IOP peak, our study was conducted between 6 and $7 \mathrm{pm}$, a time when IOP is known to be near its diurnal trough. The factors which control the circadian rhythm of IOP remain poorly understood. We speculate that the differences noted between the results of this study and previous studies may be related to factors that control IOP circadian rhythm. Further posture studies at different times of the day could potentially elucidate these differential diurnal IOP postural responses, if they exist.

Measures of CDI blood flow velocity and vascular resistance, as obtained in our study, cannot be used to quantitatively assess volumetric blood flow. Both vessel diameter and velocity are needed for this purpose. No technique is currently available to accurately and non-invasively measure retrobulbar vessel diameter. Nevertheless, data do indicate that changes in CDI velocity measures are highly predictive of changes in volumetric flow both in vitro ${ }^{13} 14$ and in vivo, in cerebral vessels. ${ }^{13} 15$ Further, considerable evidence points to a close correlation between higher resistance 
index and increased vascular resistance downstream from the point of CDI measurement. ${ }^{14}$ Consequently, our data suggest that distal vascular resistance falls in the ophthalmic artery of both normal subjects and glaucoma patients during supination. In the CRA, only normal subjects demonstrate such a change.

The internal carotid artery supplies the orbit and over $80 \%$ of the cerebrum. ${ }^{16}$ Anatomically, the ophthalmic artery exits the internal carotid artery behind the orbit and immediately bifurcates into the middle cerebral and anterior cerebral arteries. Data from the middle cerebral artery demonstrate a small non-significant change in blood flow velocity during posture change. ${ }^{2}{ }^{3}$ This result is unlike our ophthalmic artery data, which demonstrate a significant increase in velocity during supination. It appears from these data that the major feeding vessel of the brain (that is, middle cerebral artery) is more tightly autoregulated than the major feeding vessel of the eye (that is, ophthalmic artery), even though these arteries are in very close anatomical proximity. Results from other studies support this conclusion. Data show that middle cerebral artery blood velocity remains constant as blood pressure rises during isometric exercise. ${ }^{17}$ On the contrary, ophthalmic artery blood velocity increases significantly during similar conditions. ${ }^{18}$ Further, mild hypercapnia induces significant elevation of middle cerebral artery blood flow velocity, while elevated end tidal carbon dioxide appears to have no effect on the ophthalmic artery blood flow. ${ }^{19}$ If the autoregulation of the ophthalmic artery is less stringent than that of cerebral vessels, then this suggests that the eye may be more susceptible to vascular insult than the brain during periods of circulatory stress.

The CRA directly feeds and is the only source of blood supply for the retinal arteries. These distal vessels nourish the retinal ganglion cells and the confluence of unmyelinated nerve fibres anterior to the lamina cribrosa. ${ }^{20}$ Glaucoma is a disease known to cause the death of retinal ganglion cells and erosion of the optic nerve head. Numerous studies indicate that glaucoma patients have altered retinal circulation. Extensive morphological studies describe endothelial proliferations in the retinal vessels of glaucoma patients. ${ }^{21} 22$ Further, widespread angiographic circulatory defects appear in the retinal vasculature of open angle glaucoma patients ${ }^{23}$ and these patients demonstrate significantly increased arteriovenous passage time. ${ }^{24}{ }^{25}$ Such defects could potentially increase vascular resistance in the retinal vessels and, in turn, limit the autoregulatory response to posture change in retrobulbar vessels of these patients. Our data here, taken in conjunction with these previous studies, suggest that the failure to properly regulate CRA blood flow during posture change may be related to dysfunctional retinal circulation in glaucoma patients.

A number of previous studies have demonstrated that ocular pulsatility falls during supination in normal subjects ${ }^{26} 27$ and in patients with chronic open angle glaucoma, ${ }^{27}$ ocular hypertensive, ${ }^{26}$ and normal tension glaucoma. ${ }^{28}$ Ocular pulsatility is related to arterial pressure and reflects the influx of blood into the eye with each heart beat; the greater the pulse amplitude, the greater the bolus of blood entering the eye. ${ }^{29}$ The previous pulsatility findings have been interpreted to suggest that during supination, ocular perfusion falls, increasing the potential for tissue hypoxia. ${ }^{26}$ This interpretation is contrary to the implications of our results. A fall in distal vascular resistance in the retrobulbar vessels, when changing from seated to supine posture, suggests an increase (or minimally no change), not a decrease in ocular perfusion during supination.

One limitation of the pulsatility measurement is that it evaluates only the pulse wave in the eye (systole) and is virtually insensitive to the steady state non-pulsatile component (diastole) of blood flow. ${ }^{29}$ Accordingly, pulsatile blood flow and volumetric blood flow do not always move in concordance. Shifts in blood flow from the systolic to diastolic component of the cardiac cycle can alter pulsatile flow without changing volumetric flow, or vice versa. For example, when perfusion pressure is reduced in cerebral vessels due to increased intracranial pressure, pulsatile blood flow increases in the major feeding vessel (that is, internal carotid artery), while volumetric blood flow falls in this vessel. ${ }^{30}$ Further, during hypoxic hypercapnia, cerebral volumetric blood flow increases substantially $(>50 \%)$ with no corresponding change in pulsatile blood flow. ${ }^{31}$ If overall ocular vascular resistance is falling during supination, as suggested by the CDI data here, then the reduction in ocular pulsatility during supination shown in previous studies may reflect not a reduction in overall ocular perfusion but simply a shift in the blood flow away from systole to diastole. ${ }^{28}$

In summary, unlike the middle cerebral artery, Doppler measures of blood flow velocity in the ophthalmic artery change significantly during posture variation. This is true for both glaucoma patients and normal subjects. While the ophthalmic artery response is similar between groups, differences in measures of central retinal artery resistance index are not. These data indicate that posture change exposes a vascular autoregulatory deficit in glaucoma patients, which appears to be most prominent in the vessels distal to the central retinal artery.

\footnotetext{
1 Jelnes R. Twenty-four hour blood flow in the forefoot after reconstructive vascular surgery. Ann Surg 1986;204:176-80. 2 Daffertshofer M, Diehl RR, Ziems GU, et al. Orthostatic changes of cerebral blood flow velocity in patients with changes of cerebral blood flow velocity in patients

3 Briebach T, Fisher PA. Circulation studies and transcranial Doppler sonography in orthostatic regulation disorders. Ultaschall in der Medizin 1988;9:223-6.

4 Flammer J, Gasser P, Prunte $\mathrm{CH}$, et al. The probable involvement of factors other than intraocular pressure in the pathogenesis of glaucoma. In: Drance SM, Van Buskirk EM, Neufeld A, eds. Pharmacology of glaucoma. Baltimore: Williams and Wilkins, 1992:273-83.

5 Harris A, Sergott RC, Spaeth GL, et al. Color Doppler analysis of ocular vessel blood velocity in normal-tension glaucoma. Am f Ophthalmol 1994;118:642-9.

6 Butt Z, O’Brien C, McKillop G, et al. Color Doppler imaging in untreated high- and normal-pressure open-angle glaucoma. Invest Ophthalmol Vis Sci 1997;38:690-6.

7 Rankin SJA, Walman BE, Buckley AR, et al. Color Doppler imaging and spectral analysis of the optic nerve vasculature in glaucoma. Am f Ophthalmol 1995;119:685-93.
} 
8 Harris A, Williamson T, Martin B, S et al. Test/retest reproducibility of color Doppler imaging assessment of blood

9 flow velocity in orbital vessels. F Glaucoma 1995;4:281-6. imaging of the eye and orbit. Technique and normal vascular anatomy. Arch Ophthalmol 1991;109:527-31.

10 Kothe A. The effect of posture on the intraocular pressure and the pulsatile ocular blood flow in normal and glaucomatous eyes. Surv Ophthalmol 1994;38(suppl): S191-7.

11 Zeimer RC. Circadian variations in intraocular pressure. In: Ritch R, Shields MB, Krupin T, eds. The glaucomas. Vol 1. St Louis: CV Mosby 1989:319-35.

12 Buguet A, Py P, Romanet JP. 24-Hour (nyctohemeral) and sleep-related variations of intraocular pressure in healthy white individuals. Am 7 Ophthalmol 1994;117:342-7.

13 Hansen N, Stonestreet B, Rosenkrantz T, et al. Validity of Doppler measurements of anterior cerebral artery blood flow velocity: correlation with brain blood flow in piglets. Pediatrics 1983;72:526-31.

14 Spencer J, Giussani D, Moore P, et al. In vitro validation of Doppler indices using blood and water. $\mathcal{F}$ Ultrasound Med 1991;10:305-8

15 Rosenberg A, Narayanan V, Jones M. Comparison of anterior cerebral artery blood flow velocity and cerebra blood flow during hypoxia. Pediatr Res 1985;19:67-70.

16 Rhoades R, Tanner G. Medical physiology. New York: Little, Brown, 1995.

17 Rogers HB, Schroeder TV, Secher NH, et al. Cerebral blood flow during static exercise in humans. F Appl Physiol 1990; 68:2358-61.

18 Beck D, Harris A, Evans D, et al. Ophthalmic artery hemodynamics during isometric exercise. 7 Glaucoma 1995;4: $317-21$.

19 Harris A, Tippke S, Sievers C, et al. Acetazolamide and $\mathrm{CO}_{2}$ : acute effects on cerebral and retrobulbar hemodynamics. F Glaucoma 1996;5:39-45.
20 Onda E, Cioffi G, Bacon D, et al. Microvasculature of the human optic nerve. Am f Ophthalmol 1995;120:92-102.

21 Dryden RM. Central retinal vein occlusion and chronic simple glaucoma. Arch Ophthalmol 1965;73:659-63.

22 Green WR, Chan CC, Hutchins GM, et al. Central retinal vein occlusion. Trans Am Ophthalmol Soc 1981;79:371-422.

23 Schwartz B. Circulatory defects of the optic disk and retina in ocular hypertension and high pressure open-angle glaucoma. Surv Ophthalmol 1994;38(suppl):S23-33.

24 Wolf S, Arend O, Sponsel E, et al. Retinal hemodynamics using scanning laser ophthalmoscopy and hemorheology in chronic open angle glaucoma. Ophthalmology 1993;100: 1561-6.

25 Duijm F, Van Den Berg JTP, Greve E. A comparison of retinal and choroidal hemodynamics in patients with primary open-angle glaucoma and normal-pressure glaucoma. Am f Ophthalmol 1997;123:644-56.

26 Trew DR, Smith SE. Postural studies in pulsatile ocular blood flow. I. Ocular Hypertension and normotension. $\mathrm{Br} F$ Ophthalmol 1991;75:66-70.

27 Trew DR, Smith SE. Postural studies in pulsatile ocular blood flow. II. Chronic open angle glaucoma. $\mathrm{Br} \mathcal{F}$ Ophthalmol 1991;75:71-5.

28 James CB, Smith SE, Pulsatile ocular blood flow in patients with low tension glaucoma. Br F Ophthalmol 1991;75:46670 .

29 Langham ME, Farrell RA, O’Brien C. Estimation of pulsatile ocular blood flow from intraocular pressure. Acta Ophthalmol 1989;191(Suppl):9-13.

30 Nornes H, Aaslid R, Lindegaard K. Intracranial pulse pressure dynamics in patients with intracranial hypertension. Acta Neurochir 1977;38:177-86.

31 Curran-Everett D, Zhang Y, Jones RH, et al. Hypoxia, hypercapnia and hyptertension: their effects on pulsatile cerebral blood flow. F Appl Physiol 1995;79:870-8. 